\title{
Improved Weed Control Methods in Maize (Zea mays L.) By Using Single Herbicides and Their Combinations at Reduced Rates with Mineral Oil Adjuvant
}

\author{
Aref, W.M. ${ }^{1}$; R.A. Dawood $^{2}$; Anaam H. Galal ${ }^{2}$ and Z.R. Yehia ${ }^{1}$ \\ ${ }^{1}$ Weed Research Central Laboratory, Agricultural Research Center, Giza, Egypt. \\ ${ }^{2}$ Agronomy Department, Faculty of Agricultural, Assiut University, Egypt.
}

Received on: $11 / 5 / 2017$

Accepted for publication on: 17/5/2017

\begin{abstract}
:
A field experiment was carried out in Mallawy Agricultural Research Station, Agricultural Research Center, El-Minia Governorate, during 2015 and 2016 seasons to evaluate the efficacy of certain single herbicides at full rate and their mixtures at reduced rate (50\% of full rate) with mineral oil on weed control and maize productivity. Each experiment comprised of fourteen treatments as follow: the first five treatments were pendimethalin, s-metolachlor, acetochlor, nicosulfuron and dicamba+topramizonused as single herbicidesat full rate $682.5,672,840$, 24 and $63 \mathrm{~g}$ a.i./fed., respectively, the next six treatments were the sequential application of the same previous herbicides at half of the rate plus mineral oil at 1 $\% \mathrm{v} / \mathrm{v}$ and the last three treatments were (foramsulfuron sodium $3.35 \%+$ iodosulfuron-methyl sodium 0.11 + thiencarbazone-methyll1.07) used at full rate $22.65 \mathrm{~g}$ a.i./fed., hoeing twice and untreated treatment as control. Randomized Complete Block Design (RCBD) with four replications was used. The major weeds were Euphorbia geniculata, Ortega, Xanthium strumarium L., Corchorus olitorius L. and Ipomoea eriocarpa L. as broad-leaved weeds and Echinochloa colonum L. and Brachiaria reptans $\mathrm{L}$. as grasses weeds.
\end{abstract}

The findings of this study showed that the sequential application of two herbicides with different site of action (pendimethalin or s-metolachlor or acetochlor combination with nicosulfuron or dicamba+topramizon) at reduced rate plus mineral oil at $1 \% \mathrm{v} / \mathrm{v}$ gave better result in controlling broad-leaved and total weeds than applying the same previous herbicides alone at full rate, while, pendimethalin and s-metolachlor alone at full rate were the best in controlling grasses weeds.

The best total weed control efficiency (91.95 and 91.5\%) was achieved under treatment hoeing twice which was equal statistically with foramsulfuron sodium + iodosulfuron - methyl sodium + thiencarbazone - methyl (91.48 and $90.05 \%)$ and with acetochlor mixture with nicosulfuron at reduced rate plus mineral oil (87.19 and $85.37 \%$ ) controlling effect as compared with untreated treatment at first survey in both seasons, respectively. Moreover, those treatments also gave the significantly higher grain yield (28.14, 27.20 and $24.98 \mathrm{ardab} / \mathrm{fed}$.) and (26.90, 25.44 and $23.36 \mathrm{ardab} / \mathrm{fed}$. in the both seasons, respectively), as well as ear grains weight and 100-grain weight as compared with untreated treatment.

Keywords: Maize, weed control, herbicides, reduced rate, mineral oil.

\section{Introduction:}

Maize (Zea mays L.) is one of the most important among the cereal crops in the world's agricultural economy. Maize grains are used for human consumption, feed for poultry 
and livestock, for extraction of edible oil and also for starch and glucose industry.

Weeds infestation is one of the serious pests that interfere with maize crop through competition and allelopathy resulting in direct loss to quantity and quality of the product. (Pannacci and Onofri, 2016) found that crop yield losses in the untreated treatment compared to the highest obtained yield with herbicides treatments, ranging from 33 to $91 \%$. Other researchers mentioned that maize yield losses caused by weed competition have been amounted by $50 \%$ (Abouziena et al. 2013) and 33.7\% (Saudy, 2013).

Weeds control in maize fields is, very essential for obtaining good yield. Different weed control methods have been used to manage the weeds, but mechanical and chemical methods are more frequently used for the weeds control than any other control methods. Mechanical methods including hand weeding and hoeing are still useful but are getting expensive; laborious and time - consuming. Chemical weed control is a better supplement to conventional methods and forms an integral part of the modern crop because it is cheaper, faster and it gives better control.

Previous studies shown that use of currently single herbicides at the full rates did not give satisfactory results to weeds control, especially if weeds community has many and different species, whereas many of them containing active ingredient is activity only on some weed species without other, have relatively short time of action and provide only a narrow spectrum weed control (Amin et al.
2008). As that continuous usage of same herbicides or similar herbicides have same site of action year after year over several years caused changing weed flora, poor controlling and promoted the evolution of herbicides resistant weed biotypes. Hence, choice of mixtures two or more active ingredients for weed control generally increases herbicide efficacy (Pannacci et al. 2007) and (Sulewska et al. 2012). This is due to provide a broad spectrum of the target weed species and slowdown selection of herbicide-resistant weed biotypes (Zollinger, 2011).

Post-emergence herbicides must overcome a variety of barriers to their entry into plants in order to be effective. For example, herbicides applied to foliage must remain on the leaf instead of beading up and rolling off, then get past the leaf hairs and waxes on the leaf surface, then finally penetrate through the cell walls and cell membranes (DiTomaso, 1999). An adjuvant is any compound that is added to a herbicide formulation or tank mix to facilitate the mixing, application, or effectiveness of that herbicide. Adjuvants may also improve a herbicide's efficacy (Costa et al. 2005) so that the concentration or total amount of herbicide required to achieve a given effect is reduced (Naddem et al. 2008). Mineral oil adjuvant include paraffinic oil which can soften wax, or cause cracks in the cuticle, allowing increased herbicide penetration (Green, 2001).

Keeping in view the above mentioned thoughts, it was hypothesized that herbicides mixtures at reduced rate could be effect when applied with adjuvant; therefore, present 
study was, objective to evaluate the efficacy of certain single herbicides at full rate and their mixtures at half of the rate; tank mix with mineral oil 1 $\%$ on weed control and maize productivity.

\section{Materials and Methods:}

A field experimentwas carried out in Mallawy Agricultural Research Station, Agricultural Research Center, El Minia Governorate, during 2015 and 2016 seasons, each trial comprised of fourteen treatments as follow:

1. Pendimethalin $[\mathrm{N}-(1-$ ethylpropyl)-3, 4-dimethyl-2, 6 dinitrobenzeneamine] Known commercially as "Stomp extra $45.5 \%$ SC" used at full rate 682.5 $\mathrm{g}$ a.i./fed. applied after sowing before irrigation as pre - emergence (PRE).

2. $\mathrm{S}-$ metolachlor $[\mathrm{A}$ mixture of (S)-2-chloro-N-(2-ethy 1-6-methylphenyl)-N-(2-methoxy1 -methylethyl) acetamide and (R)2-chloro-N-(2-ethyl-6methylphenyl)-N-(2-methoxy-1methylethyl) acetamide] known commercially as "Dual gold $96 \%$ EC" used at full rate $672 \mathrm{~g}$ a.i./fed. applied as pre-emergence.

3. Acetochlor[2-chloro-N(ethoxymethyl)-N-(2-ethyl-6methylphenyl)acetamide]known commercially as "Harness $84 \%$ EC" used at full rate $840 \mathrm{~g}$ a.i./fed. applied as pre-emergence.

4. Nicosulfuron $[2-[[[[[(4$, 6-dimethoxy-pyrimidinyl) amino] carbonyl] amino] sulfonyl]-N, Ndimethyl-3-pyridinecarboxamide] known commercially as "Active 6 $\% \mathrm{SC}$ " used at full rate $24 \mathrm{~g}$ a.i. / fed. applied at 4-6 leaves stage of maize as post - emergence (POST).

5. Dicamba $16 \%[3,6-$ dichloro-2-methoxybenzoic acid. $]+$ Topramezon $5 \%[(3-(4,5-$ dihydro-3-isoxazolyl)-2-methyl-4(methylsulfonyl) phenyl) (5hydroxy-1-methyl-1 $H$-pyrazol-4yl) methanone)] known commercially as "Stellar star $21 \%$ SL" used at full rate $63 \mathrm{~g}$ a.i./fed. applied as post-emergence.

6. Stomp extra used at 50 $\%$ reduced rate $341.25 \mathrm{~g}$ a.i./fed. as PRE+Active at $50 \%$ reduced rate $12 \mathrm{~g}$ a.i./fed. as POST tankmixed with mineral oil known commercially as Super misrona 94\% EC added to the spray tank at $2 \mathrm{~L} /$ fed. $(1 \% \mathrm{v} / \mathrm{v})$ of the total spray volume.

7. Stomp extra at $341.25 \mathrm{~g}$ a.i. /fed. as PRE + Stellar star at $31.5 \mathrm{~g}$ a.i./fed. as POST tankmixed with mineraloil at $1 \%$.

8. Dual gold at $336 \mathrm{~g}$ a.i./fed as PRE + Active at $12 \mathrm{~g}$ a.i./fed. as POST tank-mixed with mineraloil at $1 \%$.

9. Dual gold at $336 \mathrm{~g}$ a.i./fed as PRE + Stellar star at $31.5 \mathrm{~g}$ a.i./fed. as POST tankmixed with mineraloil at $1 \%$.

10. Harness at $420 \mathrm{~g}$ a.i./fed as PRE +Active at $12 \mathrm{~g}$ a.i./fed. as POST tank-mixed with mineraloil at $1 \%$.

11. Harness at $420 \mathrm{~g}$ a.i./fed as PRE +Stellar star at $31.5 \mathrm{~g}$ a.i./fed. as POST tank-mixed with mineraloil at $1 \%$.

12. Foramsulfuron sodium $3.35 \%$ [2-[[[[(4, 6-dimethoxy-2pyrimidinyl) amino] carbonyl] 


amino]
(formylamino)- $N$,
dimethylbenzamide]+ $\begin{array}{r}\text { Iodosulfu- } \\ \text { ron-methyl sodium } 0.11 \%\end{array}$ -
[methyl 4-iodo-2-[[[[(4-methoxy-
6-methyl-1,3,5-triazin-2yl)amino]
carbonyl] amino] sulfonyl] benzo-
ate, sodium salt] + Thiencarba-
zone-methyl 1.07 \% [methyl 4-
[[[(4,5-dihydro-3-methoxy-4-
methyl-5-oxo-1H-1,2,4-triazol-1-
yl) carbonyl] amino] sulfonyl]-5-
methyl-3-thiophenecarboxylate]
known commercially as "Maister
power 4.53\% OD" used at full
rate 22.65 g a.i./fed. applied as
POST.

13. Hoeing twice at 20 and 40 days after sowing.

14. Untreated (control).

The experimental field was well prepared and calcium super phosphate $\left(15.5 \% \mathrm{P}_{2} \mathrm{O}_{5}\right)$ was applied during soil preparation at the rate of 200 $\mathrm{kg} /$ fed. Randomized Complete Block Design (RCBD) with four replicates was used. Each plot area was $10.5 \mathrm{~m}^{2}$ (5 rows X $3.0 \mathrm{~m}$ length). The row-torow and plant-to-plant distanceswere $70 \mathrm{~cm}$ and $25 \mathrm{~cm}$, respectively. The maize cultivar "single cross 128" (Zea mays L.) was used in both seasons. Maize grains were sowing manually on one side in hills at the rate of $10 \mathrm{Kg} / \mathrm{fed}$. during the $1^{\text {st }}$ week of June in both seasons. The seedlings were thinned to one plant per hill before the $1^{\text {st }}$ irrigation. Nitrogen and potassium fertilizers were applied in the form of urea $(46 \% \mathrm{~N})$ and potassium sulphate $\left(48 \% \mathrm{~K}_{2} \mathrm{O}\right)$ at the rate of $120 \mathrm{~kg} \mathrm{~N} / \mathrm{fed}$. and $50 \mathrm{~kg}$ $\mathrm{K}_{2} \mathrm{O} / \mathrm{fed}$, respectively, in two equal doses before the $1^{\text {st }}$ and $2^{\text {nd }}$ irrigations. Herbicides were applied after sowing and before irrigation as pre emergence and at 3-6 leaves stage of maize as post-emergence using "Knapsack hand sprayer CP3 20 litter" equipped with one nozzle even flat fan calibrated to deliver spray volume of $200 \mathrm{~L} /$ fed. Harvested was done on the $1^{\text {st }}$ week of October in the both seasons. The experimental field had been in a corn - Sugar beet (Beta vulgaris L.) rotation cycle for the last two years. All other agronomic practices for growing maize crop were remained constant and uniform for all treatments, except those under study.

Mechanical and chemical analysis of the soil experimental site showed that the soil texture was silty clay loam and containing 21.3, 8.43 and $186 \mathrm{ppm}$ for $\mathrm{N}, \mathrm{P}$ and $\mathrm{K}$, respectively.

\section{Collected data:}

\section{A. Weed survey:}

The weed species from one square meter chosen at random from each plot were hand pulled out and identified at the species level using the weed identification manual of (Täckholm 1974), then separated into two groups i.e. broad-leaved and grasses weeds. Thereafter, weeds were air-dried for three days and then were oven dried at $70^{\circ} \mathrm{C}$ for $48 \mathrm{hr}$., until a constant weight was reached. The dry weight of each group $\left(\mathrm{g} / \mathrm{m}^{2}\right)$ was recorded. Weed survey was carried out twice i.e. after 60 and 90 days after sowing. Weed control efficiency (WCE) was calculated as follow: $\mathrm{WCE}=\mathrm{DWC}-\mathrm{DWT} / \mathrm{DWC} \mathrm{x}$ 100. Where, DWC = Dry weight of weeds from control plot and DWT = Dry weight of weeds from treated plot. 


\section{B. Maize data:}

At harvest, random samples of ten guarded maize plants were taken from the middle three rows of each plot to estimate the following traits: Ear grains weight g, 100-grains weight (g). And grain yield ar$\mathrm{dab} /$ feddan (1ardab =140 kg) was calculated based on weight grains yield obtained from each plot, the weight were adjusted to $15.5 \%$ moisture content.

\section{Statistical analysis:}

All data were statistically analyzed according to technique of Analysis of Variance (ANOVA) for the randomized complete block design with four replications as mentioned by (Gomez and Gomez 1984), using "SAS" computer software package (SAS, 1999). Duncan's Multiple Range Test (DMRT) was used for compare among treatment means (Duncan 1955). Weed dry weight were square root transformed $(\sqrt{x+0.5})$ prior to analysis to correct for normal distribution. Data in tables are reported as non- transformed (original data).

\section{Results and Discussion:}

A. Effect of weed control treatments on weeds:-

1. Effect of weed control treatments on broad-leaved weed controlat first survey (60 days after maize sowing).

Table 1 show the effect of various weed control treatments on the dry weight of broad-leaved weeds accompanied by their equivalent weed control efficiency at first survey in 2015 and 2016 seasons. Significant effect of different studied treatments on the dry weight of broad-leaved weeds was observed as compared with untreated treatment. Presented data indicated that all studied treatments gave more than 60.92 and $52.51 \%$ weed control efficiency in 2015 and 2016 seasons, respectively, as compared with untreated (control).

The maximum broad-leaved weeds control efficiency was observed under treatment of hoeing twice 93.96 and $94.32 \%$ in the first and second seasons, respectively, which was statistically at bar with foramsulfuron sodium+iodosulfuronmethyl sodium +thiencarbazonemethyl as a ready formulated mixtures at full rate $22.65 \mathrm{~g}$ a.i./fed. which gave 93.05 and $93.66 \%$ controlling effect in the first and second seasons, respectively.

All herbicides sequential application at reduced rate $(\mathrm{PRE}+\mathrm{POST}+$ mineral oil) gave excellent control of broad-leaved weeds compared to the use of same herbicides alone at full rate without adjuvant exception of nicosulfuron alone at full rate in 2016 season.

Acetochlor or pendimethalin or s-metolachlor + nicosulfuron with mineral oil at reduced rate provided weed control efficiency by about $87.53,83.24$ and $82.31 \%$ in 2015 season and 86.66, 79.38 and $76.97 \%$ in 2016 season, respectively. Acetochlor or pendimethalin or smetolachlor+ dicamba+topramizon with mineral oil at reduced rate ranked second and provided about $73.33,71.65$ and $72.61 \%$ and 72.46 , 70.87 and $69.93 \%$ controlling effect in $1^{\text {st }}$ and $2^{\text {nd }}$ seasons, respectively.

Among herbicides applied alone at full rate, nicosulfuron and dicamba+topramizon both being at 
par with each other show the superiority over treatments pendimethalin, s-metolachlor and acetochlor alone at full rate. The maximum dry weight of broad-leaved weeds was found under untreated treatment in the both seasons.

Similar trend of results was observed during the second survey (at 90 days after maize sowing) in both seasons as shown in Table 2.
2. Effect of weed control treatments on grasses weed control at first survey (60 days after maize sowing).

Concerning the effect of various weed control treatments on the dry weight of grasses weeds recorded at first survey in both seasons, the data in Table 1 reveal that all weed control treatments significantly reduced the dry weight of the grasses weeds, compared to untreated (control).

Table 1. Dry weight of broad-leaved, grasses and total weeds at first survey (60 DAS) as influenced by various weed control treatments.

\begin{tabular}{|c|c|c|c|c|c|c|c|c|c|c|c|c|c|c|}
\hline \multirow{4}{*}{\multicolumn{2}{|c|}{\begin{tabular}{|l|} 
\\
Treatments
\end{tabular}}} & \multirow{4}{*}{$\begin{array}{l}\text { Rate g } \\
\text { a.i. / fed. }\end{array}$} & \multicolumn{12}{|c|}{$\begin{array}{l}\text { First survey at } 60 \text { days after maize sowing on } 2015 \text { and } 2016 \\
\text { seasons. }\end{array}$} \\
\hline & & & \multicolumn{6}{|c|}{ Season 2015} & \multicolumn{6}{|c|}{ Season 2016} \\
\hline & & & \multicolumn{2}{|c|}{$\begin{array}{l}\text { Broad- } \\
\text { leaved } \\
\text { weeds }\end{array}$} & \multicolumn{2}{|c|}{$\begin{array}{l}\text { Grasses } \\
\text { weeds }\end{array}$} & \multicolumn{2}{|c|}{ Total weeds } & \multicolumn{2}{|c|}{$\begin{array}{c}\text { Broad- } \\
\text { leaved weeds }\end{array}$} & \multicolumn{2}{|c|}{$\begin{array}{l}\text { Grasses } \\
\text { weeds }\end{array}$} & \multicolumn{2}{|c|}{ Total weeds } \\
\hline & & & \begin{tabular}{|c|}
$\begin{array}{c}\text { Dry } \\
\text { weight }\end{array}$ \\
\end{tabular} & \begin{tabular}{|c|} 
WCE \\
$\%$
\end{tabular} & \begin{tabular}{|c|}
$\begin{array}{c}\text { Dry } \\
\text { weight }\end{array}$ \\
\end{tabular} & \begin{tabular}{|c|} 
WCE \\
$\%$
\end{tabular} & \begin{tabular}{|c|} 
Dry \\
weight
\end{tabular} & \begin{tabular}{|c|}
$\mathrm{WCE}$ \\
$\%$ \\
\end{tabular} & $\begin{array}{l}\text { Dry } \\
\text { eight }\end{array}$ & \begin{tabular}{|c|} 
WCE \\
$\%$
\end{tabular} & \begin{tabular}{|c|} 
Dry \\
weight
\end{tabular} & $\begin{array}{c}\text { WCE } \\
\%\end{array}$ & $\begin{array}{c}\text { Dry } \\
\text { weight }\end{array}$ & $\begin{array}{c}\mathrm{WCE} \\
\%\end{array}$ \\
\hline $\mathbf{T}_{1}$ & Pendimethalin $45.5 \%$ CS. & 682.5 & \begin{tabular}{|c|}
233.78 \\
$\mathrm{~b}$
\end{tabular} & 62.21 & $6.42 \mathrm{~cd}$ & 88.98 & $240.21 \mathrm{~b}$ & 64.52 & $293.89 \mathrm{~b}$ & 55.10 & $6.28 \mathrm{gf}$ & 90.68 & $\begin{array}{c}300.17 \\
b c\end{array}$ & 58.42 \\
\hline $\mathbf{T}_{2}$ & S - metolachlor $96 \%$ EC. & 672 & $\begin{array}{c}241.80 \\
\mathrm{~b}\end{array}$ & 60.92 & $5.62 \mathrm{~d}$ & 90.35 & $247.42 \mathrm{~b}$ & 63.45 & $310.81 \mathrm{~b}$ & 52.51 & $4.29 \mathrm{~g}$ & 93.64 & $315.09 \mathrm{~b}$ & 56.35 \\
\hline $\mathbf{T}_{3}$ & Acetochlor $84 \%$ EC. & 840 & \begin{tabular}{|c|}
$\begin{array}{c}219.58 \\
\mathrm{~b}\end{array}$ \\
\end{tabular} & 64.51 & $\begin{array}{c}12.08 \\
\text { bcd }\end{array}$ & 79.28 & $231.65 \mathrm{~b}$ & 65.78 & $238.33 \mathrm{bc}$ & 63.59 & $14.50 \mathrm{c}-\mathrm{f}$ & 78.49 & $\begin{array}{c}252.82 \\
\text { bcd }\end{array}$ & 64.98 \\
\hline $\mathbf{T}_{4}$ & Nicosulfuron 6 \% SC. & 24 & $\begin{array}{c}181.07 \\
\mathrm{~b}\end{array}$ & 70.73 & $\begin{array}{c}12.26 \\
\text { bcd }\end{array}$ & 78.96 & $193.34 \mathrm{~b}$ & 71.44 & $196.15 \mathrm{~cd}$ & 70.03 & $\begin{array}{c}15.07 \\
\text { cde }\end{array}$ & 77.63 & $\begin{array}{c}211.22 \\
\text { de }\end{array}$ & 70.74 \\
\hline $\mathbf{T}_{5}$ & Dicamba+Topramizon $21 \%$ SL. & 63 & \begin{tabular}{|c|}
187.81 \\
$\mathrm{~b}$
\end{tabular} & 69.64 & $13.88 \mathrm{bc}$ & 76.19 & $201.68 \mathrm{~b}$ & 70.21 & $209.43 \mathrm{~cd}$ & 68.00 & $20.62 \mathrm{bc}$ & 69.40 & $\begin{array}{c}230.04 \\
\mathrm{~cd}\end{array}$ & 68.13 \\
\hline $\mathbf{T}_{6}$ & $\mathbf{T}_{1}+\mathbf{T}_{4}+$ Mineral oil. & \begin{tabular}{|c|}
$341.25+12+$ \\
$2 \mathrm{~L}$ \\
\end{tabular} & \begin{tabular}{|c|}
103.69 \\
$\mathrm{~d}$
\end{tabular} & 83.24 & $8.37 \mathrm{bcd}$ & 85.64 & $112.06 \mathrm{c}$ & 83.45 & 134.95 ef & 79.38 & $9.24 \mathrm{efg}$ & 86.29 & $144.19 \mathrm{fg}$ & 80.03 \\
\hline $\mathbf{T}_{7}$ & $\mathbf{T}_{1}+\mathbf{T}_{5}+$ Mineral oil. & $\begin{array}{l}341.25+ \\
31.5+2 \mathrm{~L} \\
\end{array}$ & \begin{tabular}{|c|}
$\begin{array}{c}175.42 \\
\mathrm{~b}\end{array}$ \\
\end{tabular} & 71.65 & $8.01 \mathrm{bcd}$ & 86.25 & $183.43 \mathrm{~b}$ & 72.90 & $190.67 \mathrm{~cd}$ & 70.87 & $9.82 \mathrm{~d}-\mathrm{g}$ & 85.42 & $\begin{array}{c}200.49 \\
\text { de }\end{array}$ & 72.23 \\
\hline $\mathbf{T}_{8}$ & $\mathbf{T}_{2}+\mathbf{T}_{4}+$ Mineral oil. & $\begin{array}{c}336+12 \\
+2 \mathrm{~L} \\
\end{array}$ & \begin{tabular}{|c|}
$\begin{array}{c}109.42 \\
\mathrm{~cd}\end{array}$ \\
\end{tabular} & 82.31 & $9.02 \mathrm{bcd}$ & 84.52 & $118.45 \mathrm{c}$ & 82.50 & 150.72 de & 76.97 & $9.27 \mathrm{efg}$ & 86.24 & 159.99 ef & 77.84 \\
\hline $\mathbf{T}_{9}$ & $\mathbf{T}_{\mathbf{2}}+\mathbf{T}_{\mathbf{5}}+$ Mineral oil & $\begin{array}{c}336+ \\
31.5+2 \mathrm{~L} \\
\end{array}$ & \begin{tabular}{|c|}
$\begin{array}{c}169.46 \\
\mathrm{bc}\end{array}$ \\
\end{tabular} & 72.61 & $7.09 \mathrm{~cd}$ & 87.84 & $176.55 \mathrm{~b}$ & 73.92 & $196.83 \mathrm{~cd}$ & 69.93 & 8.65 efg & 87.16 & $\begin{array}{c}205.48 \\
\text { de }\end{array}$ & 71.54 \\
\hline $\mathbf{T}_{\mathbf{1 0}}$ & $\mathbf{T}_{3}+\mathbf{T}_{4}+$ Mineral oil. & $420+12+2 L$ & \begin{tabular}{|c|}
$\begin{array}{c}77.14 \\
\text { de }\end{array}$ \\
\end{tabular} & 87.53 & $9.58 \mathrm{bcd}$ & 83.56 & $86.73 \mathrm{~cd}$ & 87.19 & $87.30 \mathrm{f}$ & 86.66 & $18.33 \mathrm{~cd}$ & 72.79 & $\begin{array}{c}105.63 \\
\text { gh }\end{array}$ & 85.37 \\
\hline $\mathbf{T}_{11}$ & $\mathbf{T}_{3}+\mathbf{T}_{5}+$ Mineral oil. & $\begin{array}{c}420+ \\
31.5+2 \mathrm{~L} \\
\end{array}$ & $\begin{array}{c}165.00 \\
\mathrm{bc}\end{array}$ & 73.33 & $13.57 \mathrm{bc}$ & 76.72 & $178.56 \mathrm{~b}$ & 73.62 & $180.22 \mathrm{cde}$ & 72.46 & 13.74 -f & 79.60 & $\begin{array}{c}193.97 \\
\text { def }\end{array}$ & 73.13 \\
\hline $\mathbf{T}_{12}$ & $\begin{array}{l}\text { Foramsulfuro sodium + Iodosulfu- } \\
\text { ron-methyl sodium + Thiencarba- } \\
\text { zone-methyl } 4.53 \% \text { OD. }\end{array}$ & 22.65 & $\begin{array}{c}43.02 \\
\text { ef }\end{array}$ & 93.05 & $14.52 \mathrm{bc}$ & 75.09 & $57.53 \mathrm{~d}$ & 91.50 & $41.51 \mathrm{~g}$ & 93.66 & $30.35 \mathrm{~b}$ & 54.95 & $71.86 \mathrm{hi}$ & 90.05 \\
\hline$T_{13}$ & Hoeing twice at 20 - 40 DAS. & & $37.34 \mathrm{f}$ & 93.96 & $17.15 \mathrm{~b}$ & 70.57 & $54.49 \mathrm{~d}$ & 91.95 & $37.20 \mathrm{~g}$ & 94.32 & $24.34 \mathrm{bc}$ & 63.88 & $61.54 \mathrm{i}$ & 91.48 \\
\hline$T_{14}$ & Untreated (control). & & \begin{tabular}{|c|}
$\begin{array}{c}618.70 \\
\mathrm{a}\end{array}$ \\
\end{tabular} & \begin{tabular}{|l|}
0.00 \\
\end{tabular} & $58.28 \mathrm{a}$ & 0.00 & 676.97 a & 0.00 & $654.52 \mathrm{a}$ & 0.00 & $67.38 \mathrm{a}$ & 0.00 & $721.89 \mathrm{a}$ & 0.00 \\
\hline F te & est $=$ & & $* *$ & & $* *$ & & $* *$ & & $* *$ & & $* *$ & & $* *$ & \\
\hline
\end{tabular}

Any two means in the same column sharing same letters did not differ significantly by Duncan at $5 \%$ level of probability.

$\mathrm{WCE}=$ Weed control efficiency. 
Table 2. Dry weight of broad-leaved, grasses and total weeds at second survey (90 DAS) as influenced by various weed control treatments.

\begin{tabular}{|c|c|c|c|c|c|c|c|c|c|c|c|c|c|c|}
\hline \multirow{4}{*}{\multicolumn{2}{|c|}{ Treatments }} & \multirow{4}{*}{$\begin{array}{c}\text { Rate g } \\
\text { a.i. / fed. }\end{array}$} & \multicolumn{12}{|c|}{ Second survey at 90 days after maize sowing on 2015 and 2016 seasons. } \\
\hline & & & \multicolumn{6}{|c|}{ Season 2015} & \multicolumn{6}{|c|}{ Season 2016} \\
\hline & & & \multicolumn{2}{|c|}{$\begin{array}{c}\begin{array}{c}\text { Broad-leaved } \\
\text { weeds }\end{array} \\
\end{array}$} & \multicolumn{2}{|c|}{ Grasses weeds } & \multicolumn{2}{|c|}{ Total weeds } & \multicolumn{2}{|c|}{$\begin{array}{c}\text { Broad-leaved } \\
\text { weeds }\end{array}$} & \multicolumn{2}{|c|}{ Grasses weeds } & \multicolumn{2}{|c|}{ Total weeds } \\
\hline & & & \begin{tabular}{c|} 
Dry \\
weight
\end{tabular} & \begin{tabular}{|c|} 
WCE \\
$\%$
\end{tabular} & \begin{tabular}{|c|} 
Dry \\
weight
\end{tabular} & $\begin{array}{c}\mathrm{WCE} \\
\%\end{array}$ & \begin{tabular}{|c|} 
Dry \\
weight
\end{tabular} & \begin{tabular}{|c|}
$\mathrm{WCE}$ \\
$\%$
\end{tabular} & \begin{tabular}{|c|}
$\begin{array}{c}\text { Dry } \\
\text { weight }\end{array}$ \\
\end{tabular} & \begin{tabular}{|c|} 
WCE \\
$\%$
\end{tabular} & \begin{tabular}{|c|} 
Dry \\
weight
\end{tabular} & $\begin{array}{c}\mathrm{WCE} \\
\%\end{array}$ & \begin{tabular}{|c|} 
Dry \\
weight
\end{tabular} & $\begin{array}{c}\mathrm{WCE} \\
\%\end{array}$ \\
\hline $\mathbf{T}_{1}$ & Pendimethalin $45.5 \%$ CS. & 682.5 & \begin{tabular}{|c|}
477.80 \\
$\mathrm{bc}$
\end{tabular} & 54.20 & $\begin{array}{c}14.91 \\
\mathrm{de}\end{array}$ & 82.35 & \begin{tabular}{|c|}
$\begin{array}{c}492.70 \\
\mathrm{bc}\end{array}$ \\
\end{tabular} & 56.31 & \begin{tabular}{|c|}
551.96 \\
$\mathrm{c}$
\end{tabular} & 49.91 & $20.36 \mathrm{f}$ & 82.93 & \begin{tabular}{|c|}
572.32 \\
$\mathrm{c}$ \\
\end{tabular} & 53.14 \\
\hline $\mathbf{T}_{\mathbf{2}}$ & S - metolachlor $96 \%$ EC. & 672 & $\begin{array}{c}520.38 \\
\mathrm{~b}\end{array}$ & 50.11 & $11.41 \mathrm{e}$ & 86.49 & \begin{tabular}{|c|}
531.79 \\
$\mathrm{~b}$
\end{tabular} & 52.84 & $\begin{array}{c}646.36 \\
\mathrm{~b}\end{array}$ & 41.34 & $18.41 \mathrm{f}$ & 84.57 & $\begin{array}{c}664.77 \\
\mathrm{~b}\end{array}$ & 45.57 \\
\hline $\mathbf{T}_{3}$ & Acetochlor & 840 & $\begin{array}{c}437.47 \\
\text { bcd }\end{array}$ & 58.06 & \begin{tabular}{|c|}
$23.26 \mathrm{~b}-$ \\
$\mathrm{e}$
\end{tabular} & 72.46 & \begin{tabular}{|c|}
$\begin{array}{c}460.73 \\
\text { bcd }\end{array}$ \\
\end{tabular} & 59.14 & $\begin{array}{c}487.55 \\
\mathrm{~cd}\end{array}$ & 55.75 & $\begin{array}{c}37.08 \\
\text { cde }\end{array}$ & 68.92 & \begin{tabular}{|c|}
$\begin{array}{c}524.63 \\
\mathrm{~cd}\end{array}$ \\
\end{tabular} & 57.04 \\
\hline $\mathbf{T}_{4}$ & Nicosulfi & 24 & $\begin{array}{c}392.1 \\
\text { bcd }\end{array}$ & 62.41 & $\begin{array}{c}29.43 \\
b c\end{array}$ & 65.16 & \begin{tabular}{|c|}
$\begin{array}{c}421.56 \\
\text { bcd }\end{array}$ \\
\end{tabular} & 62.61 & $\begin{array}{c}44.10 \\
\text { def }\end{array}$ & 59.70 & $\begin{array}{c}42.39 \\
\text { cde }\end{array}$ & 64.47 & \begin{tabular}{|c|}
$\begin{array}{c}486.49 \\
\text { cde }\end{array}$ \\
\end{tabular} & 60.16 \\
\hline $\mathbf{T}_{5}$ & Dicamba+Top & 63 & $\begin{array}{c}404.91 \\
\text { bcd }\end{array}$ & 61.18 & $\begin{array}{c}21.49 \\
\text { cde }\end{array}$ & 74.55 & \begin{tabular}{|c|}
$\begin{array}{c}426.40 \\
\text { bcd }\end{array}$ \\
\end{tabular} & 62.19 & $\begin{array}{c}455.71 \\
\mathrm{de}\end{array}$ & 58.64 & $\begin{array}{c}40.29 \\
\text { cde }\end{array}$ & 66.24 & \begin{tabular}{|c|}
$\begin{array}{c}496.00 \\
\text { cde }\end{array}$ \\
\end{tabular} & 59.39 \\
\hline $\mathbf{T}_{6}$ & $\mathbf{T}_{1}+\mathbf{T}_{4}+$ Mineral oil. & \begin{tabular}{|c|}
$341.25+$ \\
$12+2 \mathrm{~L}$ \\
\end{tabular} & $\begin{array}{c}324.19 \\
\mathrm{~d}\end{array}$ & 68.92 & $\begin{array}{l}19.68 \\
\text { cde }\end{array}$ & 76.70 & \begin{tabular}{|c|}
343.87 \\
$\mathrm{~d}$
\end{tabular} & 69.50 & $\begin{array}{c}352.20 \\
\mathrm{~g}\end{array}$ & 68.04 & $\begin{array}{c}29.79 \\
\text { def }\end{array}$ & 75.03 & $381.99 \mathrm{f}$ & 68.72 \\
\hline $\mathbf{T}_{7}$ & $\mathbf{T}_{1}+\mathbf{T}_{5}+$ Miner & \begin{tabular}{|l|}
$341.25+$ \\
$31.5+2 \mathrm{~L}$ \\
\end{tabular} & $\begin{array}{c}374.99 \\
\mathrm{~cd}\end{array}$ & 64.05 & $\begin{array}{c}25.67 \\
\text { bcd }\end{array}$ & 69.61 & \begin{tabular}{|c|}
$\begin{array}{c}400.66 \\
\mathrm{~cd}\end{array}$ \\
\end{tabular} & 64.47 & $\begin{array}{c}403.61 \\
\mathrm{~d}-\mathrm{g}\end{array}$ & 63.37 & $\begin{array}{c}6.45 \\
\text { cde }\end{array}$ & 69.45 & $\begin{array}{c}440.06 \\
\text { def }\end{array}$ & 63.97 \\
\hline $\mathbf{T}_{8}$ & $\mathbf{T}_{2}+\mathbf{T}_{4}+$ Mineral oil. & $\begin{array}{c}336+ \\
12+2 \mathrm{~L}\end{array}$ & $\begin{array}{c}348.44 \\
\mathrm{~d}\end{array}$ & 66.60 & $\begin{array}{l}19.46 \\
\text { cde }\end{array}$ & 76.96 & $\begin{array}{c}367.90 \\
\text { cd }\end{array}$ & 67.37 & $\begin{array}{c}62.89 \\
\text { fg }\end{array}$ & 67.07 & $\begin{array}{c}26.80 \\
\text { ef }\end{array}$ & 77.54 & $389.69 \mathrm{f}$ & 68.09 \\
\hline $\mathbf{T}_{9}$ & $\mathbf{T}_{2}+\mathbf{T}_{5}+$ Mineral oil & \begin{tabular}{|c|}
$336+$ \\
$31.5+2 \mathrm{~L}$ \\
\end{tabular} & $\begin{array}{c}374.57 \\
\text { cd }\end{array}$ & 64.09 & $\begin{array}{c}17.20 \\
\text { cde }\end{array}$ & 79.64 & \begin{tabular}{|c|}
$\begin{array}{c}391.77 \\
\mathrm{~cd}\end{array}$ \\
\end{tabular} & 65.26 & $\begin{array}{c}430.89 \\
\text { d-g }\end{array}$ & 60.90 & $\begin{array}{c}26.95 \\
\text { def }\end{array}$ & 77.41 & \begin{tabular}{|c|}
$\begin{array}{c}457.85 \\
\text { def }\end{array}$ \\
\end{tabular} & 62.51 \\
\hline $\mathbf{T}_{10}$ & $\mathbf{T}_{3}+\mathbf{T}_{4}+$ Mineral oil. & $\begin{array}{l}420+ \\
12+2 \mathrm{~L}\end{array}$ & $\begin{array}{c}197.79 \\
\mathrm{e}\end{array}$ & 81.04 & $\begin{array}{c}21.13 \\
\text { cde }\end{array}$ & 74.98 & \begin{tabular}{|c|}
218.92 \\
$\mathrm{e}$
\end{tabular} & 80.59 & $\begin{array}{c}211.69 \\
\mathrm{~h}\end{array}$ & 80.79 & $\begin{array}{c}44.36 \\
\text { cd }\end{array}$ & 62.82 & $\begin{array}{c}256.05 \\
\mathrm{~g}\end{array}$ & 79.03 \\
\hline$T_{11}$ & $\mathbf{T}_{3}+\mathbf{T}_{5}+$ Mineral oil. & \begin{tabular}{|c|}
$420+$ \\
$31.5+2 \mathrm{~L}$ \\
\end{tabular} & $\begin{array}{c}346.28 \\
\mathrm{~d}\end{array}$ & 66.80 & $\begin{array}{l}18.36 \\
\text { cde }\end{array}$ & 78.26 & \begin{tabular}{|c|}
364.64 \\
$\mathrm{~cd}$
\end{tabular} & 67.66 & $\begin{array}{c}\begin{array}{c}395.33 \\
\text { efg }\end{array} \\
\end{array}$ & 64.12 & $\begin{array}{c}26.96 \\
\text { def }\end{array}$ & 77.41 & $\begin{array}{c}422.28 \\
\text { ef }\end{array}$ & 65.42 \\
\hline$T_{12}$ & $\begin{array}{l}\text { Foramsulfuro sodium + Iodo- } \\
\text { sulfuron-methyl sodium }+ \\
\text { Thiencarbazone-methyl } 4.53 \\
\% \text { OD. }\end{array}$ & 22.65 & $\begin{array}{c}157.86 \\
\text { ef }\end{array}$ & 84.87 & $38.17 \mathrm{~b}$ & 54.80 & $\begin{array}{c}196.03 \\
\text { ef }\end{array}$ & 82.62 & $145.79 \mathrm{i}$ & 86.77 & $69.51 \mathrm{~b}$ & 41.74 & $\begin{array}{c}215.31 \\
\mathrm{gh}\end{array}$ & 82.37 \\
\hline $\mathbf{T}_{13}$ & $\begin{array}{l}\text { Hoeing twice at } 20-40 \\
\text { DAS. }\end{array}$ & & $100.30 \mathrm{f}$ & 90.38 & $38.25 \mathrm{~b}$ & 54.71 & $138.55 \mathrm{f}$ & 87.71 & $120.69 \mathrm{i}$ & 89.05 & $\begin{array}{c}52.00 \\
\mathrm{bc}\end{array}$ & 56.42 & $\begin{array}{c}172.69 \\
\mathrm{~h}\end{array}$ & 85.86 \\
\hline $\mathbf{T}_{14}$ & Untreated (control). & & \begin{tabular}{|c|}
1043.14 \\
$\mathrm{a}$ \\
\end{tabular} & 0.00 & $84.46 \mathrm{a}$ & 0.00 & \begin{tabular}{|c|}
1127.60 \\
$\mathrm{a}$
\end{tabular} & 0.00 & \begin{tabular}{|c|}
1101.91 \\
$\mathrm{a}$
\end{tabular} & 0.00 & $\begin{array}{c}119.31 \\
\mathrm{a}\end{array}$ & 0.00 & \begin{tabular}{|c|}
1221.23 \\
$\mathrm{a}$
\end{tabular} & 0.00 \\
\hline \multicolumn{3}{|c|}{$F$ test $=$} & $* *$ & & $* *$ & & $* *$ & & $* *$ & & $* *$ & & $* *$ & \\
\hline
\end{tabular}

Any two means in the same column sharing same letters did not differ significantly by Duncan at $5 \%$ level of probability.

$\mathrm{WCE}=$ Weed control efficiency.

It is clear from recorded data on grasses weed control efficiency in Table 1 that all studied treatments gave 70.52 to $90.35 \%$ and 54.95 to $93.64 \%$ controlling effect in 2015 and 2016 seasons. Minimum dry weight of grasses weeds was recorded in treatment s-metolachlor 5.62 and $4.29 \mathrm{~g} / \mathrm{m}^{2}$ with 90.35 and $93.64 \%$ and pendimethalin 6.42 and $6.28 \mathrm{~g} / \mathrm{m}^{2}$ with 88.98 and $90.68 \%$ controlling effect both alone at full rate as compared with untreated treatment which gave higher weight 58.28 and 67.38 $\mathrm{g} / \mathrm{m}^{2}$ in the first and second seasons, respectively.

Use the same two herbicides at half of the rate in sequence combina- tion with other herbicides (nicosulfuron or dicamba+topramizon) at reduced rate plus mineral oil found effective, ranked second and provided about 84.52 to $87.87 \%$ and 85.42 to $87.16 \%$ controlling effect in both seasons, respectively. Moreover, the other herbicidal treatments and hoeing twice were significantly higher than the untreated (control). The minimum control against grasses weeds was found under treatment hoeing twice $17.15 \mathrm{~g} / \mathrm{m}^{2}$ with $70.57 \%$ in 2015 season and under treatment foramsulfuron sodium+iodosulfuronmethyl sodium+thiencarbazonemethyl $24.34 \mathrm{~g} / \mathrm{m}^{2}$ with $63.88 \%$ in 2016 season as compared with un- 
treated treatment. Similar trend of results was observed during the second survey (90 Days after sowing) in both seasons as shown in Table 2.

3. Effect of weed control treatments on total annual weeds control at first survey (60 days after maize sowing).

Table 1 show the effect of various chemical treatments and hoeing twice on the control of total annual weeds present at the site of application in comparison with the untreated treatment. Statistical analysis of the presented data in Table 1 shows that the dry weight of total annual weeds were significantly affected by various weed control treatments.

During the first survey the treatments of hoeing twice and foramsulfuron sodium + iodosulfuronmethyl sodium + thiencarbazonemethyl provided a consistent control of total weeds by $91.95,91.48 \%$ and $91.5,90.05 \%$ in the first and second seasons, respectively.

Applying post-emergence nicosulfuron or dicamba+topramizon at $50 \%$ reduced rate plus mineral oil in sequence with pre-emergence acetochlor or pendimethalin or smetolachlor at $50 \%$ reduced rate gave excellent results in control total annual weeds and higher than use the same herbicides alone at full rate, where, $50 \%$ acetochlor + nicosulfuron with mineral oil ranked second with 87.19 and $85.37 \%$ controlling effect in the both seasons, respectively, without significantly different from treatments hoeing twice.

Other sequential application treatmentsplusmineral oil gave good results on control total annual weeds with 72.9 to $83.45 \%$ and 71.54 to
$80.03 \%$ controlling effect in $1^{\text {st }}$ and $2^{\text {nd }}$ seasons, respectively. Postemergence nicosulfuron, dicamba+topramizon and preemergence pendimethalin, smetolachlor and acetochlor both of them alone at full rate gave adequate control of total annual weeds with 63.45 to $71.44 \%$ and 56.35 to 70.74 $\%$ controlling effect in $1^{\text {st }}$ and $2^{\text {nd }}$ seasons, respectively. Similar trend of results was observed during the second survey (90 Days after sowing) in both seasons as shown in Table 2 .

As the results of the present study are summarized, it can be suggested that hoeing twice treatment was the most effective treatment for controlling the total annual weeds associated with maize as compared with other herbicidal treatments and untreated (control). These results are in line harmony with those obtained by Sharara et al. (2005), Kandil and Kordy,(2013), Marzouk, (2013) and Saudy, (2013) who found that treatment of hoeing twice at 25 and 40 DAS was the best treatment in controlling total weeds and caused reduction in weed dry weight reaching 90.5 $\%$ compared to untreated (control).

The best chemical treatments for controlling total annual weeds without significant different with hoeing twice was achieved under treatment foramsulfuron sodium + iodosulfuron-methyl sodium + thiencarbazone-methyl at full rate $22.65 \mathrm{~g}$ a.i. /fed., since this herbicide contain three active ingredient to broad spectrum of weed control and its new formulation OD was formed with oil and can be used without adjuvant.

Obtained resulted from this study show that total weed control 
increased and was more consistent when two herbicide application (PRE + POST plus oil adjuvant) were used at reduced rate compared with a single-herbicide application (either PRE or POST) alone at full rate. Hence, use of two different chemicals with different site of action gave broad spectrum of weed control and enhanced the efficacy of weed control. In this respect, several research findings indicated that two herbicides applications increase weed control compared with a single herbicide Bogdan et al. (2007), Pannacii et al. (2007) and Amine et al. (2008). Some researchers also shown that two herbicides applications at reduced rate increase weed control compared with a single herbicide Kenezivic et al. (2003) and Sulewska et al. (2012). Also, Dogan et al.(2005), Kir and Dogan, (2009) and Sostarcic et al. (2015) shown that in the most cases effective weed control can be achieved with reduced rates of single herbicides with adjuvant addition. Idziak and Woznica, (2010) and Woznica and Idziak, (2015) who have shown that two herbicides applications at reduced rate with adjuvant increase weed control compared with a single herbicide.

The present finding from this study showed that due to at least two or more of the weed species flora in these study occur as dominating weed flora on the most of the growing maize fields in the region and none of herbicides application alone at full rate would do not provide acceptable broad spectrum weed control under this weed flora, therefore, it is important to use herbicides with different site of action in sequence at reduced rate with mineral oil adjuvant to achieve broad spectrum weed control.

B. Effect of weed control treatments on maize yield and its components:-

\section{Ear grains weight (g).}

The mentioned data in Table 3 reveal that the weed control treatments had a significant influence on ear grains weight in both seasons. All weed control treatments significantly increased ear grains weight/g over the untreated treatment.

Thus, (acetochlor+ nicosulfuron+mineral oil) at reduced rate was the best and gave 219.6 and 212.84 $\mathrm{g} /$ ear higher than other sequential application of reduced herbicides plus oil adjuvant, but the higher of all the treatments in this respect 236.9 and $234.07 \mathrm{~g} /$ ear was achieved under treatment hoeing twice which was equal statistically with foramsulfuron sodium+iodosulfuron-methyl sodium + thiencarbazone-methyl 228.55 and $222.05 \mathrm{~g} /$ ear and with the above mentioned treatment (acetochlor+ nicosulfuron+mineral oil) at reduced rate in the first and second seasons, respectively. The average increased for ear grains weight/g in those treatments as compared with untreated treatment was $123.49,115.61$ and $107.17 \%$ and $125.04,113.48$ and $104.63 \%$ in the first and second seasons, respectively.

Other treatments of sequential application of pendimethalin or smetolachlor or acetolachlor + nicosulfuron or dicamba+topramezon at reduced rate + mineral oil provided good ear grains weight over same herbicides used alone at full rate and this herbicides which used alone at 
full rate gave ear grains weight higher than untreated treatment.

These findings are in line with those observed by Sharara et al. (2005) who found that hoeing twice increase the ear grains weight by $72.00-73.92 \%$ and chemical control increased ear grains weight by 26.60 $64.00 \%$ as compared with (control). The minimum value of ear grains weight 106 and $104.01 \mathrm{~g} /$ ear was obtained under untreated treatment. These results are in confirmatory with those reported by Abouziena et al. (2013) who found that weed competition caused significant reduction in the value of ear grains weight by $50.5 \%$ than hoeing twice.

\section{100-grain weight (g).}

The data regarding 100-grain weight $g$ are presented in Table 3 perusal of the data indicated that 100grain weight was significantly affected by various studied weed control treatments.

Hoeing twice treatment produced the highest 100-grain weight $(41.51$ and $42.06 \mathrm{~g})$ followed by treatment foramsulfuron sodium + iodosulfuron-methyl sodium + thiencarbazone-methyl (40.6 and $41.48 \mathrm{~g}$ ) and treatment acetochlor+ nicosulfuron+oil mineral (40.1 and $40.26 \mathrm{~g}$ ) in the first and second seasons, respectively. The above mentioned treatments increased parameter of 100- grain weight by $39.16,36.11$ and $34.44 \%$ in 2015 season and by 42.03 , 40.07 and $35.96 \%$ in 2016 season over the untreated treatment. Moreover, the other treatments applying sequential (PRE + POST + mineral oil) at reduced rate gave 100-grain weight higher than use these herbicides alone at full rate and these two groups gave 100-grain weight higher over untreated treatment and the average increased of those above treatments for 100-grain weight as compared with untreated treatment was 14.98 to $34.44 \%$ and 13.04 to $35.96 \%$ in the first and second seasons, respectively. Saudy, (2013) found that treatment of hoeing twice was the effective practice for increasing 100-grain weight by $8.19 \%$ over control as well as Bashir et al. (2015) showed that the reduced rate of herbicides can be reduced up $50 \%$ by addition adjuvant and increase 1000grain weight of maize compared full dose without adjuvant and control. The minimum value of 100 -grains weight (29.83 and $29.61 \mathrm{~g}$ ) was obtained under untreated treatment. These results are confirmatory with those of Abouziena et al. (2013) who found that weed competition caused significant reduction in the value of 100 -grains weight / g by $8.87 \%$ than hoeing twice. 
Table 3. Effect of weed control treatments on yield and its components of maize.

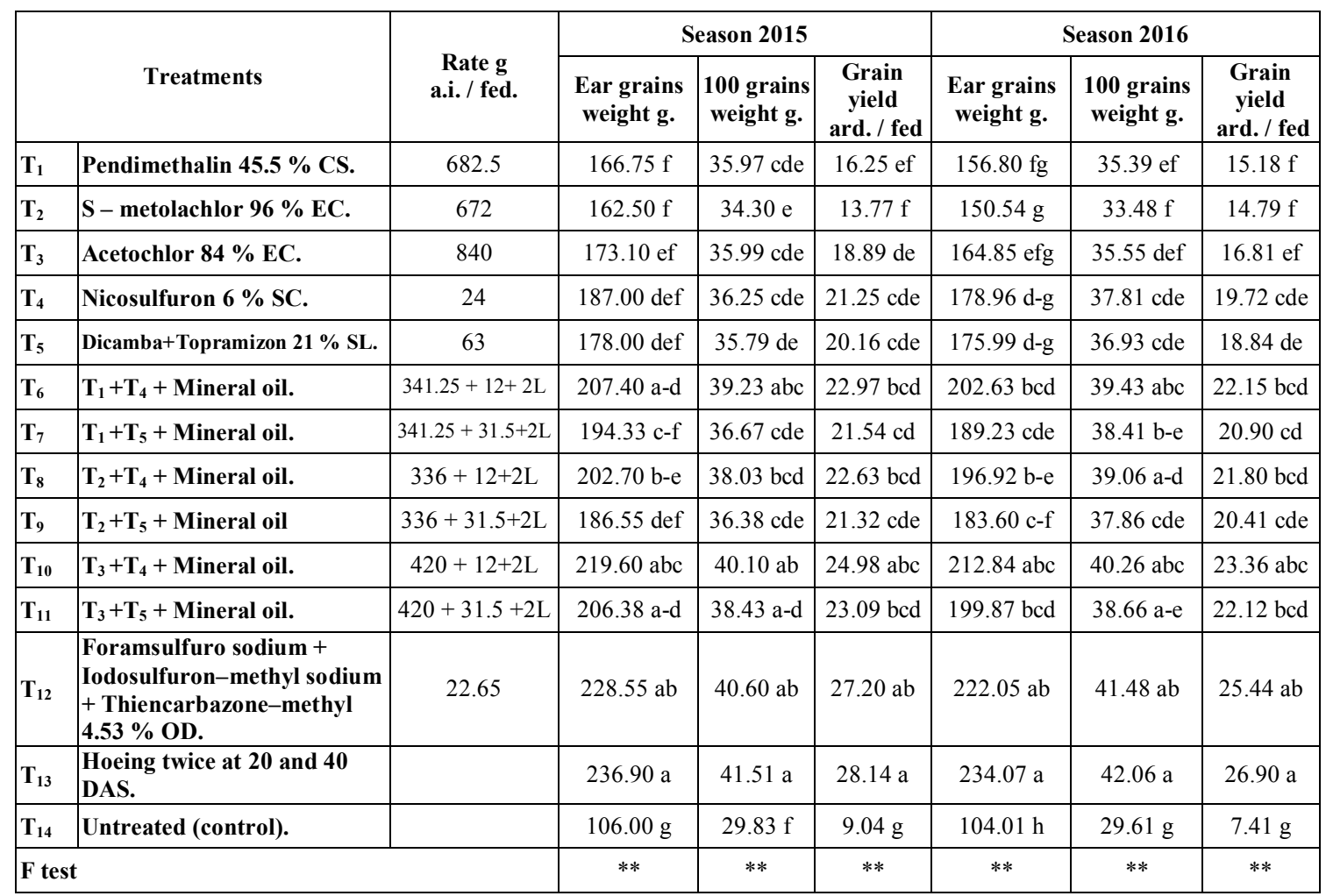

Any two means in the same column sharing same letters did not differ significantly by Duncan at $5 \%$ level of probability.

\section{Grain yield in ar- dab/feddan.}

Data regarding the effect of weed control treatments on grain yield (ardab/fed.) are shown in Table 3 which clearly indicates grain yield (ardab/fed.) to be significantly affected by weed control treatments.

The significantly higher grain yield (28.14 and 26.90 ardab/fed.) was observed under treatment hoeing twice which was equal statistically at par with foramsulfuron sodium + iodosulfuron-methyl sodium + thiencarbazone-methyl (27.2 and 25.44 $\operatorname{ardab} /$ fed.) and with treatment acetochlor+ nicosulfuron+mineral oil (24.98 and $23.36 \mathrm{ardab} / \mathrm{fed}$.). The average increased for grain yield in these treatments as compared with untreated treatment was 211.48, 201.02 and $176.48 \%$ and 263.22,
243.45 and $215.45 \%$ in the $1^{\text {st }}$ and $2^{\text {nd }}$ seasons, respectively.

There is a clear superiority of grain yield were found as a result of application herbicides in sequence combination (PRE + POST + oil mineral) at reduced rate from use the same herbicide alone at full rate. The average decreased of herbicides applied alone at full rate for grain yield ardab/fed. as compared with herbicides applied in combination sequence at reduced rate was 6.35 to 23.25 and 1.27 to $14.64 \%$ in the $1^{\text {st }}$ and $2^{\text {nd }}$ seasons, respectively. While, the herbicide whether alone at full rate or in sequence at reduced rate plus mineral oil increased the grain yield of maize by 52.38 to 155.53 and 99.69 to $198.61 \%$ as compared with untreated treatment in the $1^{\text {st }}$ and $2^{\text {nd }}$ seasons, respectively. 
Higher grain yield of maize under mechanical treatment (hoeing twice) and chemical treatments (foramsulfuron sodium + iodosulfuronmethyl sodium + thiencarbazonemethyl and acetochlor + nicosulfuron plus oil mineralmay be due to the fact effective those treatments in control of weeds and reduced competition of weeds which lead to direct increase in uptake of nutrient, water and radiation which resulted in proper growth, development and increased photosynthetic efficiency of maize plants which resulted in ear grains weight and 100-grain weight ultimately resulting into increased grain yield.

These findings are in line with those observed by Hassan and Ahmed, (2005) and Saudy, (2013) who reported that treatment of hoeing twice was the effective practice for increasing maize grain yield t/ha by $33.71 \%$. Moreover, Patel et al. (2006) and Sikkema et al. (2008) showed that mixture herbicide at different mode of action application at the $50 \%$ reduced rate versus an untreated check still allowed for increased corn yield ( 8.3 vs. $4.9 \mathrm{t} / \mathrm{ha}$ ). On the other hand, the minimum values of grain yield $(9.04$ and $7.41 \mathrm{ardab} / \mathrm{fed}$.) was obtained under untreated treatment. These results are confirmatory with those of Tesfay et al. (2014) who found that weed interference with maize crop in the treatment weedy check caused a relative yield loss by (63.66 and $75.71 \%$ ) in the both seasons, respectively, compared to the treatment of hand weeding and hoeing. Other researchers mentioned that maize yield losses caused by weed competition have been amounted by $8.87 \%$ Abouziena et al. (2013) and 33-91\% Pannacci and Onofri, (2016).

\section{Conclusion:}

Based on the findings emerged out from the present investigation, use of sequence herbicides with different site of action at reduced rate plus oil adjuvant rather than single herbicides at full rate is more effective in weed control and increased grain yield in maize. Also hoeing twice at 20 and 40 days after sowing maize was found to be effective for weed control and increased grain yield if laborers are available easily.

\section{References:}

Abouzinea, H.F., M. A. Ahmed, M. A. T. Eldabaa and M. S. A. Abd El Wahed (2013): A Comparative study on the productivity of two yellow maize cultivars grown under various weed control management. Middle East J. Agric. Res., 2(2): 56-67.

Amin A., M.A. Khan, G. Hassan, K.B. Marwat, H. Rashid and K. Nawab (2008): Weed control efficacy and economics of pre-emergence herbicides in maize (Zea mays L.). The Asian and Australasian J. of Plant Sci. and Biotechnolo. 2(2): 72-75.

Bashir, F., M.A. Nadeem, A. Tanveer and T. Abbas (2015): Impact of row spacing and reduced herbicide doses along with adjuvant on weeds in maize (Zea mays L.). Current Sci. perspectives 1(14): 112-118.

Bodgan I., P. Gus, T. Rusu, A. Pop, A. Vaju and P. Morar (2007): Research concerning weed control in maize crop. Cercetări Agronomice în Moldova, 1:129.

Costa, N. V., D. Martins, R.A. Rodella and L.D.N. de Camargo da Costa (2005): Droplet deposition during spray and leaf $\mathrm{PH}$ in aquatic weed 
control. Scientia Aggricola (62): 227-234.

DiTomaso, J.M. (1999): Barriers to foliar penetration and uptake of herbicides. Proceedings of the California Weed Sci. Society (51): 150-155.

Dogan M.N., O. Boz, and A. Unay (2005): Efficacies of reduced herbicide rates for weed control in maize (Zea mays L.) during critical period. J. Agron., 4 (1): 44-48.

Duncan, B.O. (1955): Multiple range test F. Tests. Biometercs, 11: 1-42.

Gomez, K.A. and Gomez, A.A. (1984): Statistical Procedures for Agricultural Research ( $2^{\text {nd }}$ Ed.). John Wiley and Sons, New York, $680 \mathrm{p}$.

Green, J.M. (2001): Herbicide adjuvants. In: UC Davis WRIC Weed Science School, September 26-28, 2001, Woodland, CA.

Hassan, A.A.A. and M.K.A. Ahmed (2005): The influence of some herbicides and additional hoeing in maize growth and yield components. Inst. J. Agri. Biol., 7(5):708710.

Idziak, R. and Z. Woznica (2010): Efficiency assessment of limited doses of herbicide mixtures applied with adjuvants in maize. Acta Scientiarum Polonorum- Agri. 9(4):17-28.

Kandil, E.E.E. and A.M. Kordy (2013): Effect of hand hoeing and herbicides on weeds, growth, yield and yield components of maize (Zea mays L.). J. Appl. Sci. Res., 9(4):3075-3082.

Kir K. and M.N. Dogan (2009): Weed control in maize (Zea mays L.) with effective minimum rates of foramsulfuron. Turk. J. Agric. For., 33: 601-610.

Knezevic, M., M. Durkic, I. Knezevic and Z. Loncaric (2003): Effects of pre- and post-emergence weed control on weed population and maize yield in different tillage sys- tems. Plant soil environ. 49(5): 223-229.

Marzouk A.S. (2013): Effect of some herbicides on annual grass and broad leaved weeds in maize crop: I - Effect of foramsulfuron herbicide. Bull. of Faculty of Agri., Cairo Uni.64(3):322-331.

Nadeem M.A., R. Ahmad, M. Khalid, M. Naveed, A. Tanveer and J.N. Ahmad (2008): Growth and yield response of autumn planted maize (Zea mays L.) and its weeds to reduced dose of herbicide application in combination with urea. Pak. J. Bot., 40(2): 667-676.

Pannacci E., F. Graziani and G. Covarelli (2007): Use of herbicide mixtures for pre and postemergence weed control in sunflower (Helianthus annuus). Crop Prot.26: 1150-1157.

Pannacci E. and A. Onofri (2016): Alternatives to terbuthylazine for chemical weed control in maize. Communications in Biometry and Crop Sci., 11(1): 51-63.

Patel V. J., P. N. Upadhyay, j. B. Patel and B. D. Patel (2006): Evaluation of herbicide mixtures for weed control in maize (Zea mays L.) under middle Gujarat conditions. The J. of Agri. Sci., 2(1): 81:86.

SAS Institute. (1999): SAS Systems, Version 8. Statistical Analysis Systems Institute, Cary, NC, USA.

Saudy H.S. (2013): Easily practicable packages for weed management in maize. African Crop Sci. J. 21(4):291-301.

Sharara, F.A., T.A. El-Shahawy and K.G. El-Rokiek (2005): Effect of some novel herbicides on the controlling weeds associated with maize plants. J. Agron., 4(2):88-95.

Sikkema, P. H., R. E. Nurse, T. Welacky and A. S. Hamill (2008): Reduced herbicide rates provide acceptable weed control regardless of corn 
planting strategy in Ontario field corn. Canadian J. of Plant Sci. 88(2):373-378.

Sostarcic, V., M. Scepanovic and K. Baric (2015): Effect of the reduce topramezone rate and adjuvants on Chenopodium album (L) and Echinochloa crus-galli (L) in maize. Glasilo Biljne Zastite.15 (4):255263.

Sulewska H., W. Koziara, K. Śmiatacz, G. Szymańska and K. Panasiewicz (2012): Efficacy of selected herbicides in weed control of maize. Fragm. agron. 29(3):144-151.

Täckholm, V. (1974): Students Flora of Egypt. $2^{\text {nd }}$ ed., Cairo University, Cairo, 888 p.
Tesfay A., M. Amin and N. Mulugeta (2014): Management of weeds in maize (Zea mays L.) through various pre and post emergency herbicides. Adv. Crop Sci. Tech. 2 (5):100051.

Woznica, Z. and R. Idziak (2015): Influence of reduced rates of herbicides applied with adjuvants at various dates on weed control and yield of maize. Fragmenta Agronomica. 32(2):111-118.

Zolliger, R. K. (2011): North Dakota weed control guide. 135 p. North Dakota University (NDSU) Extension Service, Fargo, North Dakota, USA. 
طرق مكافحة الحشائش المحسنة فى الذرة الثامية بإستخدام المبيدات المنفردة وتوليفاتها

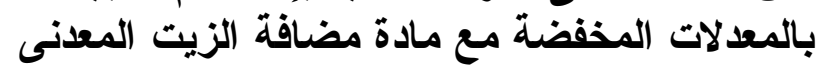

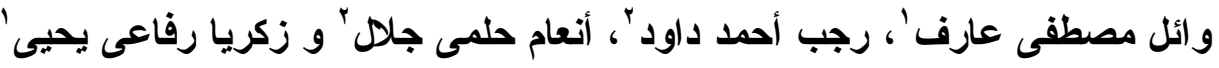

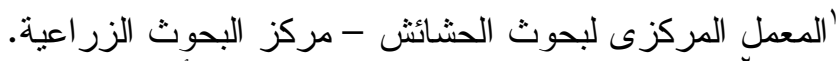
"آقسم المحاصيل - كلية الزراعة - جامعة أسيوط.

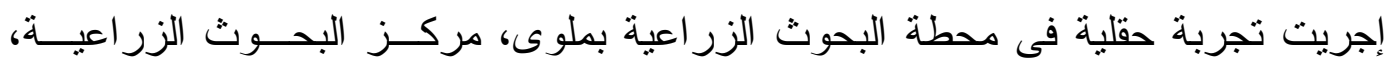

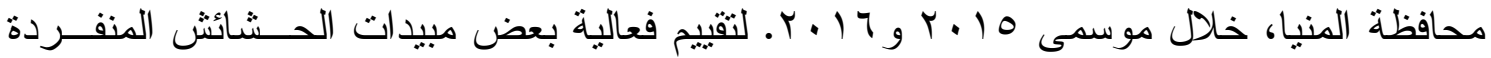

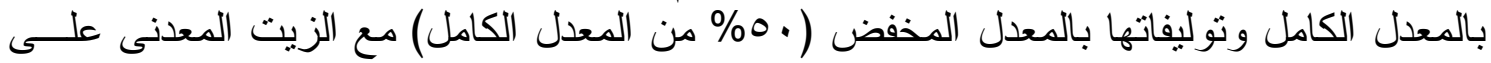

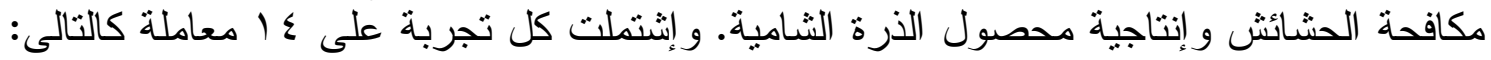

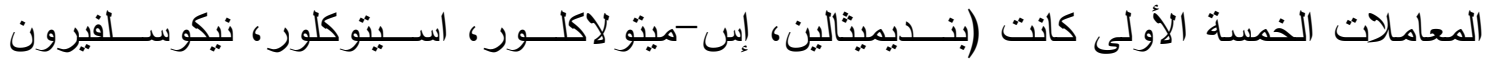

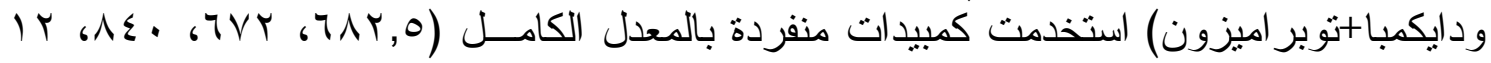

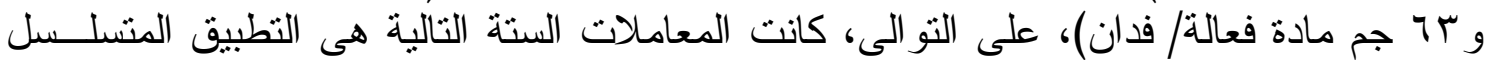

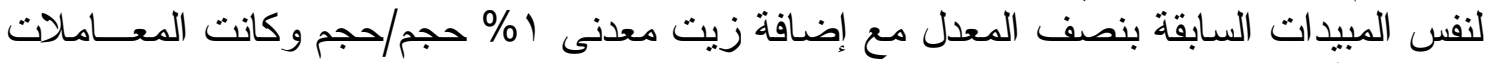

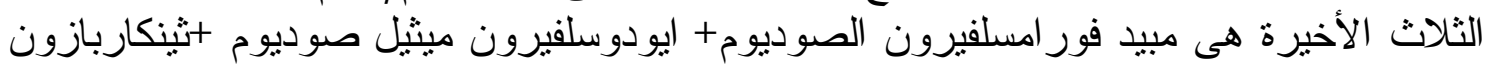

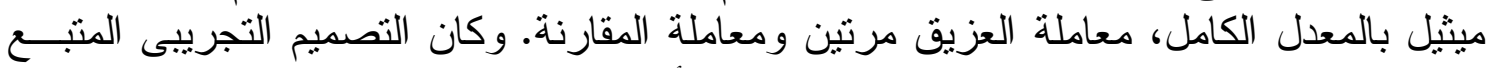

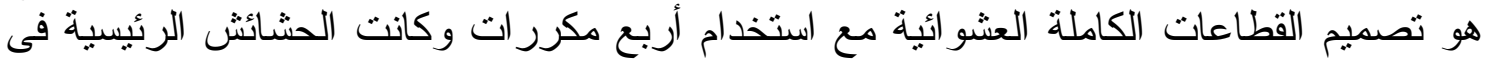

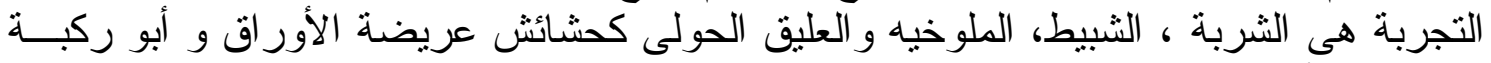
وحشيشة الأر انب كحشائش نجيلية.

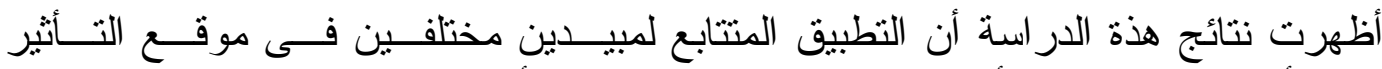

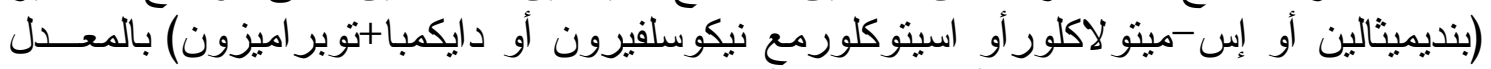

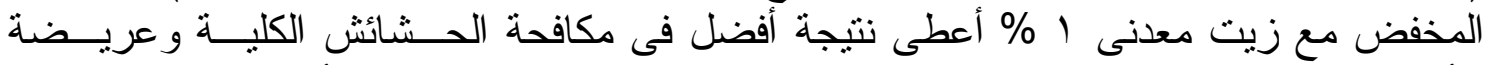

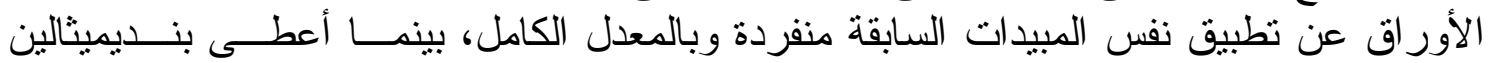

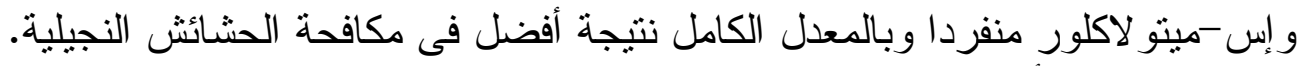

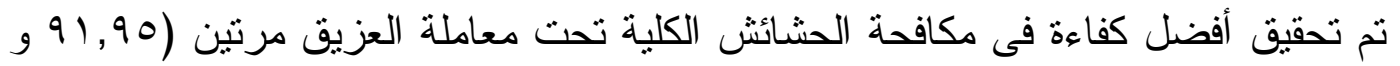

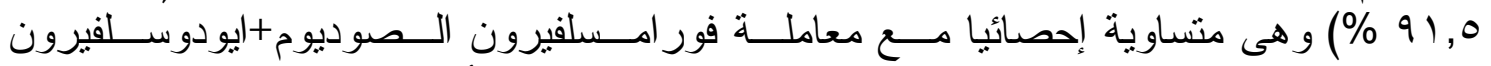

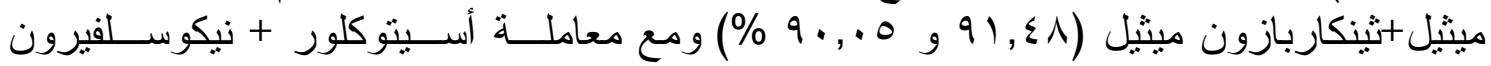

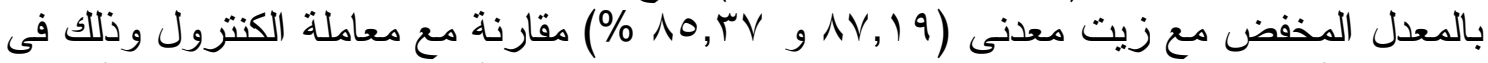

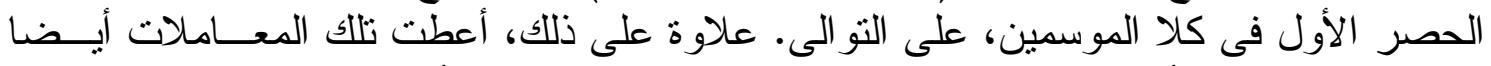

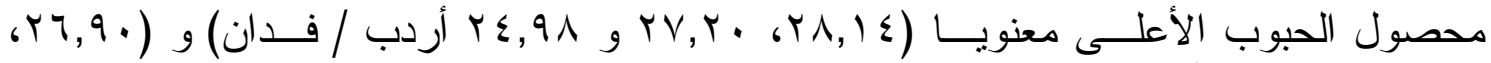

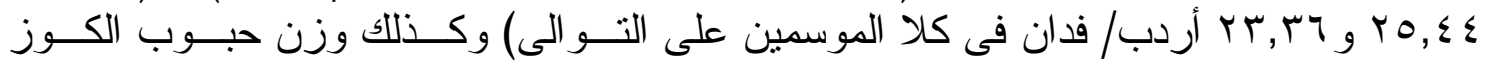

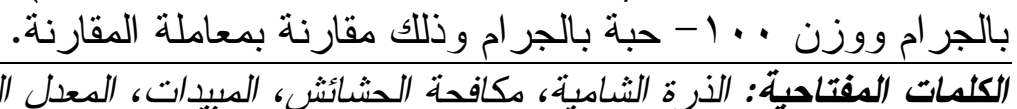

